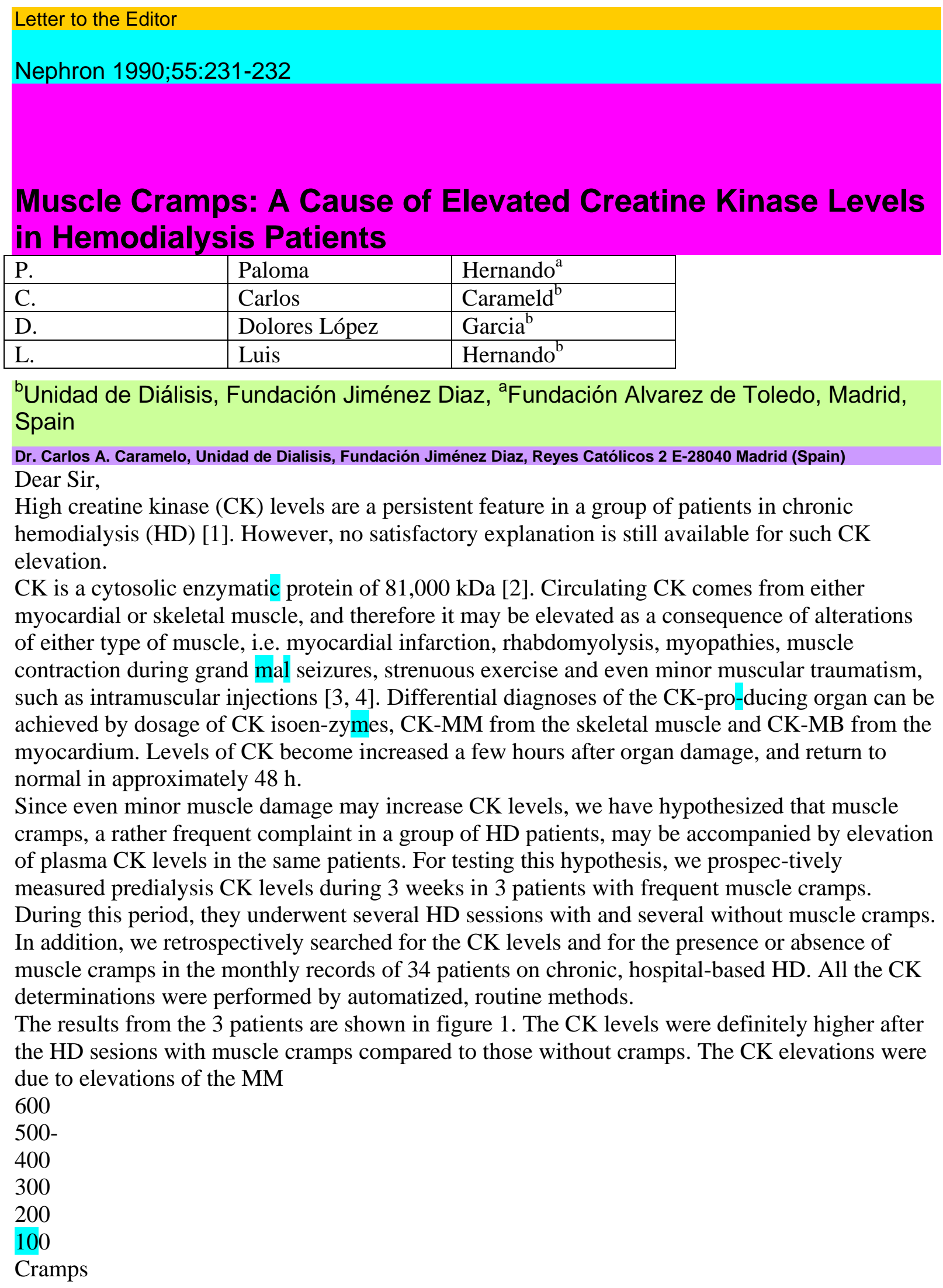




\section{No cramps}

Fig. 1. The figure represents CK values in 3 patients undergoing HD. The values on the left correspond to HD sessions with no cramps, whereas the values of the right correspond to HD sessions with cramps. Values are mean for the HD sessions of 3 weeks.

isoenzyme, and no changes in the MB isoenzyme were detected, the CK-MB values being less than $3 \%$ of the total CK in all the instances [data not shown]. Such CK-MB levels can be considered nonsignificantly elevated [4,5]. On the other hand, by searching in the monthly records of the patients undergoing treatment in the dialysis unit, three groups of individuals were identified (table 1): first, one group without cramps, which have normal to low CK values (group 1); second, a group of patients with cramps and increased CK levels (group 2), and third, a group of patients with cramps but without elevated CK (group 3). The group 2 had the highest CK values in the months in which cramps were present, as

232

Hernando/Caramelo/LópezGarcia/Hernando

compared to months without cramps. No significant differences were found between the three groups of patients in several clinical and analytical parameters, including sex, body weight, type of kidney disease, type of dialyzer, blood creatinine, electrolytes, $\mathrm{C} 03 \mathrm{H}$, total plasma proteins and P. However, the patients of the group with cramps and CK elevations were significantly younger than of the other two groups (group $244.5 \pm 3.9$ vs. group $158.7 \pm 2.52$ vs. group 3 $54.5 \pm 3.8$; $\mathrm{p}<0.05$ ), suggesting that a relationship may exist between the amount of muscle mass and muscular activity and the CK increase. Furthermore, group 2 presented lower values of plasma Ca $\left(9.12 \pm 25^{*}\right.$ vs. $9.82 \pm 38$ vs. $\left.9.44 \pm 23,{ }^{*} \mathrm{p}<0.05\right)$ and higher values of plasma ferritin $\left(1,483 \pm 399 *\right.$ vs. $986 \pm 464$ vs. $\left.380 \pm 126,{ }^{*} p<0.05\right)$ than the other two groups. No differences in the number or intensity of muscle cramps could be recalled retrospectively from the patient records. Even though no increased CK levels were detected in any patient without muscle cramps, there were patients with cramps who did not present CK elevation (table 1). No explanation is available for the latter finding, although undetected differences in the intensity, duration or metabolic characteristics of the muscle cramps in each particular patient may be the effective reason. Any one of the aforementioned factors may be critical in determining the level of CK release from the muscle [6]. Some role of the extracellular Ca is suggested by the reduction of plasma Ca observed in the patients with higher CK levels, although the actual physiological significance of such data is uncertain. In the same regard, the increased ferritin levels in the patients with highest CK levels suggest that there may exist in those patients some degree of myopathy secondary to iron deposition, thus conditioning an increased CK release from muscles. These aspects require further investigation, to ascertain whether they are pathogenetic mechanisms of the CK elevation in dialysis patients.

In summary, the present observations identify a subset of dialysis patients having elevations of CK circulating levels, in relation with the occurrence of intradialytic muscle cramps. This may contribute to clarifying the pathogenetic mechanisms of the so far unexplained CK elevation in individuals undergoing HD.

Acknowledgments

The authors wish to acknowledge Dr. J. Nuño and Dr. J.A. Gonzalez Azpeitia for the CK determinations.

References 
Vaziri ND, Kim I: Serum enzyme levels; in Daugirdas JT, Ing TS (eds): Handbook of Dialysis. Boston, Little, Brown, 1988, pp 316-320.

Kuby SA, Nodal C, Lardy HA: Adenosine-triphosphate-cre-atine transphosphorylase. J Biol Chem 1954;209:191-201.

Colombo JP, Richterich R, Rossi U: Serum Kreatin-Phosphoki-nase. Bestimmung und diagnostische Klin Wochenschr. 1962;40:37-44.

Bruns DE: Diagnosis of acute myocardial infarction when skeletal muscle damage is present: A caveat regarding use of creatine kinase isoenzymes. Clin Chem 1989;35:705.

Arenas J, Diaz V, Liras G, et al.: Activities of creatine kinase and its isoenzymes in serum in various skeletal and muscle disorders. Clin Chem 1988;34:2460-2462. 